\title{
Chip-integrierte photonische Bauelemente
}

\author{
Patrick Steglich*, Claus Villringer, Silvio Pulwer, Birgit Dietzel, Viachaslau Ksianzou und Sigurd Schrader
}

\section{Zusammenfassung}

In unserer hochtechnologisierten Gesellschaft spielt die optische Datenübertragung aufgrund der stetig wachsenden Informationsvielfalt eine immer bedeutendere Rolle. In den Anfängen der Nachrichtentechnik waren Datenraten von wenigen bit/s realisierbar. Heute werden mittels optischer Technologien Übertragungsraten von mehreren Gbit/s umgesetzt. Möglich wird dies durch neue Entwicklungen in der Chip-integrierten Photonik. Beispiele dafür sind Chip-integrierte elektrooptische Modulatoren und Schalter. In diesem Artikel werden neue Entwicklungen in der Chip-integrierten Photonik diskutiert und die experimentelle Charakterisierung der Bauelemente in Form eines Ringresonators beschrieben. Für die Experimente wird exemplarisch ein photonisches Bauelement genutzt, das aus einem hybriden SiliziumPolymer-Materialsystem beseteht. Die Ergebnisse zeigen, dass diese Materialkombination vielversprechend für zukünftige Chip-integrierte photonische Bauelemente mit extrem geringem Energiebedarf ist.

\section{Abstract}

The focus on high-tech in our society makes optical data transmission increasingly important due to the continually growing diversity of information. At the very beginning of integrated photonics, the data rates achieved were only a few bit/s. Today, transfer rates of several Gbit/s are possible due to novel chip-integrated devices such as electro-optical modulators and switches. This trend was made possible due to new developments in the field of Chip-integrated photonics. In this article, we discuss latest developments in the field of chip-integrated photonic devices and describe their experimental characterization. The experimental setup is developed and described in detail. The example used in our experiments is a hybrid silicon-polymer material system. Our results show that the hybrid material approach is a promising candidate for future on-chip integrated photonic devices with low power consumption.

\section{Einführung}

In dem Artikel „Integrated Optics: An Introduction" formulierte Miller im Jahre 1969 erstmals die Idee, optische Komponenten zu miniaturisieren und in Analogie zur Elektronik verschiedene Komponenten auf einem gemeinsamen Substrat zu integrieren (Miller 1969). Diese integrierten optischen Schaltkreise basieren auf optischen Wellenleitern und funktionellen Komponenten. Beispiele für funktionelle Komponenten sind elektrooptische Modulatoren und Schalter, die in Abhängigkeit von einer Spannung das Licht modulieren bzw. schalten.

Elektrooptische Modulatoren haben sich für Langstrecken (long-haul) und Mittelstrecken (z. B. Metropolitan Area Networks) als Signalwandler von elektrischen Signalen in Lichtsignale etabliert. Die Integration von elektro- optischen Modulatoren als aktiver Teil für die Übertragung optischer Signale im Kurzstreckenbereich (Local Area Networks, FTTH - Fibre To The Home, Rack-to-Rack, Board-to-Board), aber auch für die integrierte Optik (Chipto-Board, On-Chip) sind Entwicklungsziele der nächsten Jahre. Aufgrund der technologisch ausgereiften, zuverlässigen und kostengünstigen CMOS-Technologie (CMOS - Complementary Metal Oxide Semiconductor) gelten elektrooptische Modulatoren auf der Basis der Siliziumtechnologie als aussichtsreiche Kandidaten für Massenprodukte mit potentiell hoher Marktdurchdringung.

Bandbreiten und Übertragungsgeschwindigkeiten optischer Signale in siliziumbasierten Modulatoren sind jedoch aufgrund geringer und verlustbehafteter optischer Nichtlinearitäten von Silizium auf einen Bereich von ei- nigen zehn Gbit/s beschränkt. Ursache dafür sind die relativ geringe Ladungsträgerbeweglichkeit und die hohen Ladungsträgerlebensdauern bei dissipativen (resonanten) Prozessen wie der resonanten Zweiphotonenabsorption oder der Absorption durch freie Ladungsträger.

\section{Neue Entwicklungen in der Chip- integrierten Photonik}

Heute werden Chip-integrierte Komponenten aus Silizium, funktionellen Polymeren oder aus einer Kombination beider Materialien hergestellt. Letzteres hat sich in den vergangenen Jahren durch die Kombination der Vorteile beider Materialeigenschaften und die Entwicklung von neuartigen organischen Materialien für elektrooptische Anwendungen immer mehr etabliert und wird im Folgenden als 
Silizium-Organik-Hybrid-(SOH)-Photonik bezeichnet (Korn et al. 2014). Zur Erhöhung der Übertragungsgeschwindigkeit ist es notwendig, alternative Materialien einzusetzen, die kürzere inhärente Schaltzeiten für elektrische bzw. optische Signalübertragungsvorgänge aufweisen und höherwertige digitale Modulationsarten wie die Quadraturamplitudenmodulation (QAM) ermöglichen. Dazu ist eine gleichzeitige, voneinander unabhängige Amplituden- und Phasenmodulation nötig. Im Fall von Halbleitermaterialien wie Silizium ist dies wegen der resonanten Anregungsprozesse nicht realisierbar. Deshalb sind organische Materialien von besonderem Interesse, welche hohe dispersive (off-resonante) optische Nichtlinearitäten aufweisen, weil mit innen Amplitude, Phase und ggf. die Polarisation optischer Felder unabhängig voneinander beeinflusst werden können und diese Materialien daher für die QAM prädestiniert sind. Die SOH-Technologie nutzt die Vorteile der etablierten CMOS-Technologie, um neuartige integrierte photonische Bauelemente zu schaffen, in welche organische Materialien mit besonderen linearen und nichtlinearen optischen Eigenschaften implementiert sind. Eine wichtige Variante der SOH-Photonik nutzt mikro- und nanostrukturierte Siliziumsubstrate mit vorgefertigten Bauelementstrukturen, in welche die organischen Funktionsmaterialien mit geeigneten Depositionsmethoden an speziellen Bereichen, wie beispielsweise der Kavität eines Fabry-PerotInterferometers oder Ringresonators, eingebracht werden.

Neue Entwicklungen im Bereich des Bauelementdesigns im Bereich der $\mathrm{SOH}-P h o t o n i k$ führten zudem zu einem großen Interesse in der Forschung. Durch die Optimierung der Wellenleiterstrukturen gelang es, die Vorteile organischer Materialien zu nutzen und gleichzeitig auf etablierte Herstellungsverfahren der Halbleitertechnologie zurückzugreifen (Steglich et al. 2015b, 2015c). So konnte kürzlich ein effektiver optischer Schalter auf der Basis einer Siliziumtechnologie hergestellt werden, der zusätzlich elektrooptische Polymere neben dem Silizium als aktives Material nutzt (Steglich et al. 2015a, 2016a, 2016b). Nicht nur die Bauelementdesigns, son-
Siliziumwellenleiter

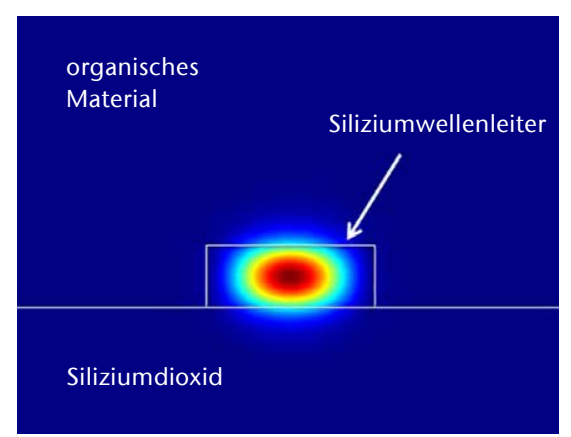

Schlitzwellenleiter

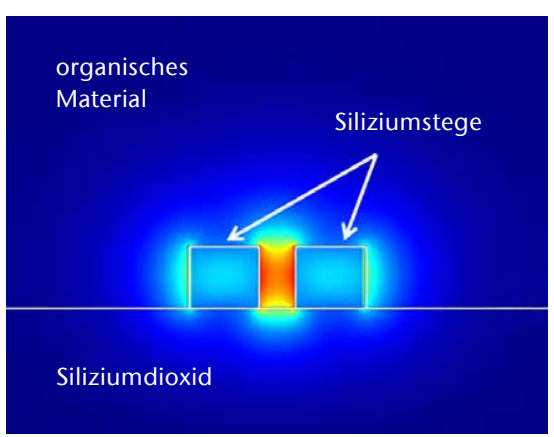

Abb. 1) Querschnitt durch einen herkömmlichen Siliziumwellenleiter (links) und dessen Feldverteilung im Vergleich zu einem Schlitzwellenleiter (rechts). Die Feldüberhöhung im Schlitzwellenleiter ermöglicht eine effiziente Nutzung der optischen Nichtlinearität des im Schlitzwellenleiter deponierten organischen Funktionsmaterials (Steglich et al. 2015c). dern auch elektrooptische Polymere konnten in den letzten Jahrzehnten weiterentwickelt werden und erreichen mittlerweile lineare elektrooptische Koeffizienten von bis zu 230 pm/V (Dalton 2010). Die Polymere dienen als Cladding, also als Mantelmaterial, und werden dementsprechend direkt auf den Siliziumwellenleiter deponiert.

Abb. 1 zeigt zwei verschiedene Wellenleiterarten, den Kanalwellenleiter und den Schlitzwellenleiter. Sie stellt vergleichend das optische Feld dar, welches von einem gewöhnlichen Siliziumwellenleiter bzw. einem Schlitzwellenleiter geführt wird. Dabei bilden Hochindexkontrastwellenleiter, wie Schlitzwellenleiter auch genannt werden, das Schlüsselbauelement zur Implementierung organischer Materialien in die Silizium-Photonik. Von Vorteil ist hierbei die Eigenheit von Schlitzwellenleitern, dass ein Großteil des Lichts im Schlitz zwischen den beiden Siliziumstegen des Wellenleiters geführt wird, also dort, wo sich auch Folge ist eine höhere Interaktion des geführten Lichts und des aktiven Polymers.

Der Grund für die Lichtverstärkung im Schlitz ist folgender: Der hohe Brechungsindexkontrast zwischen Silizium $(\mathrm{n}=3,48)$ und dem organischen Material ( $n=1,3$ bis 1,8 ) verursacht eine hohe Diskontinuität der optischen Feldstärkeamplituden an den Grenzdas organische Material befindet. Die

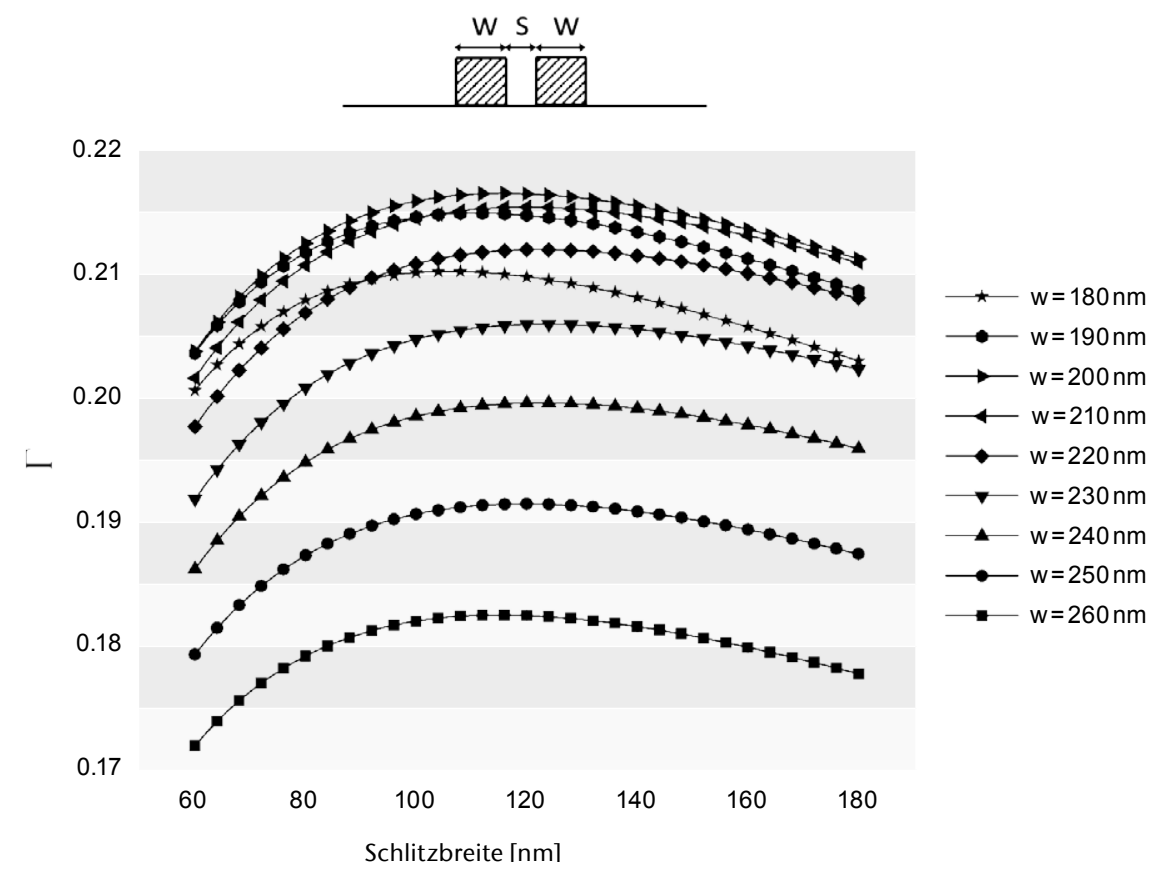

Abb. 2) Simulierter Field Confinement Factor eines Schlitzwellenleiters in Abhängigkeit von der Schlitzbreite und der Stegbreite als Parameter (Steglich et al. 2015c). 
flächen zwischen beiden Materialien, was zu einer starken Konzentration des im Schlitz geführten Lichts führt. Ein Maß für die Lichtkonzentration ist der sogenannte Field Confinement Factor. Der Field Confinement Factor

$$
\Gamma=\frac{\iint_{\text {Schlitz }} \operatorname{Re}\left(\vec{E} \cdot \vec{H}^{*}\right) d A}{\iint_{\text {Total }}^{\text {met }} \operatorname{Re}\left(\vec{E} \cdot \vec{H}^{*}\right) d A}
$$

wird definiert als Verhältnis der im Zeitmittel im Schlitz transferierten Leistung zur zeitlich gemittelten Leistung, welche insgesamt im System übertragen wird.

Abb. 2 zeigt die Ergebnisse einer Simulationsstudie für eine vorgegebene Lichtwellenlänge von 1550 nm. Dargestellt ist der Field Confinement Factor in Abhängigkeit von der Schlitzbreite $s$ und der Stegbreite w.

Nicht nur Wellenleiterarten sind in den letzten Jahren entstanden, auch photonische Bauelemente wie Ringresonatoren wurden weiterentwickelt. Ringresonatoren, basierend auf Schlitzwellenleitern mit elektronischen Kontakten, besitzen kleine QFaktoren, auch Gütefaktoren genannt. Der Gütefaktor ist die Gesamtenergie des im Resonator geführten Lichts in Bezug auf den auftretenden Energieverlust pro Umlauf. Resonatoren für optische Modulatoren benötigen Gütefaktoren von 10.000 bis 30.000 , was mit Resonatoren, auf Schlitzwellenleitern basieren, nicht möglich ist.

Bisherige Resonatoren, basierend auf Schlitzwellenleitern mit elektronischen Kontakten, besitzen Gütefaktoren von bis zu 5.000 (Gould et al. 2011). Grund dafür ist, dass die elektronischen Kontakte den Q-Faktor reduzieren, da sie zu höheren optischen Verlusten aufgrund von Absorption durch freie Ladungsträger führen. Die elektronischen Kontakte sind allerdings notwendig, um das geführte Licht aktiv schalten zu können. Ein weiterer Grund für die kleinen Gütefaktoren sind die hohen optischen Verluste an den kleinen Ringradien. Mit dem an der TH Wildau entwickelten Konzept des teilweise geschlitzten Resonators lassen sich diese Verluste verringern, so dass Gütefaktoren von 10.000 bis 100.000 erreicht worden sind (Steglich et al. 2015a).

Das neue Konzept besteht darin, dass ein Schlitzwellenleiter mit herkömmlichen Siliziumwellenleitern kombiniert wird. Dies führt zu einer Reduktion optischer Verluste an den kritischen Stellen, wie an den erwähnten Ringradien. Diese Kombination erlaubt eine Erhöhung der Übertragungsgeschwindigkeit bzw. der Sensitivität eines entsprechenden optischen Sensors. Ein weiterer erfinderischer Schritt des neuen Konzepts liegt in der
Nutzung eines sogenannten Strip-toSlot-Mode-Converters, durch welchen man Licht von der einen zur anderen Lichtwellenleiterart mit geringen optischen Verlusten einkoppeln kann. Diese Lichtkonverter wurden direkt als Teil des Resonators implementiert und nicht, wie bisher üblich, vor dem Resonator platziert (Steglich et al. 2016a).

\section{Experimentelle Charakterisierung Chip-integrierter photonischer Bauelemente}

Für die optische Charakterisierung der photonischen Bauelemente wurde ein Messplatz aufgebaut, der es erlaubt, sowohl passive als auch aktive Messungen vorzunehmen. Abb. 4 zeigt den gesamten Aufbau und eine Vergrößerung der Faserkopplung mit der das Licht in den Chip eingekoppelt wird. Mit passiven Messungen sind optische Transmissionsmessungen gemeint. Als aktive Messungen werden die Transmissionsmessungen bei gleichzeitigem Anlegen einer elektrischen Spannung, die einen elektrooptischen Effekt hervorruft und so das Transmissionsverhalten des Ringresonators beeinflussen. Für die Messungen wird eine SuperlumineszenzDiode oder ein durchstimmbarer Laser verwendet, deren Licht durch Koppelgitter in den Siliziumwellenleiter einund wieder ausgekoppelt wird.

Zur Justage der optischen Fasern wer-
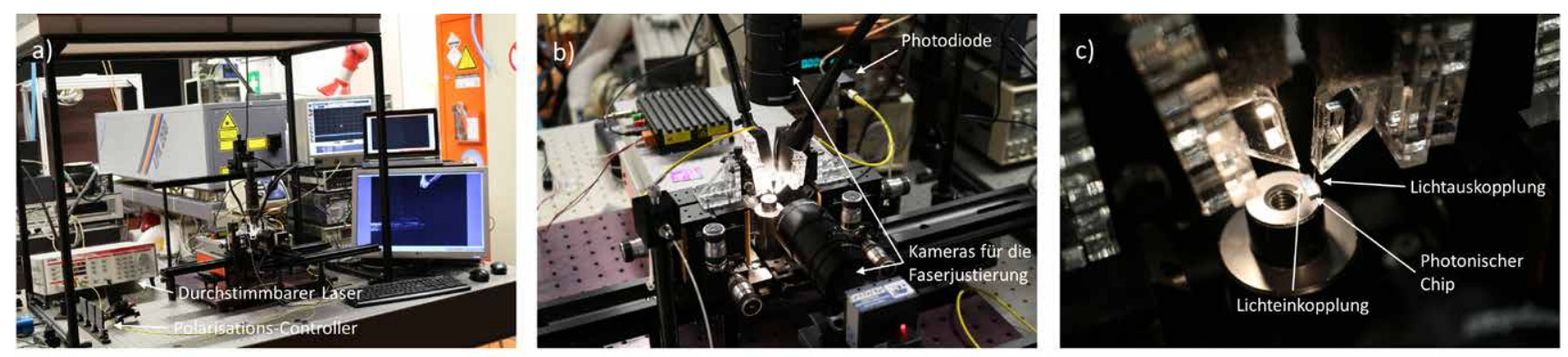

d)

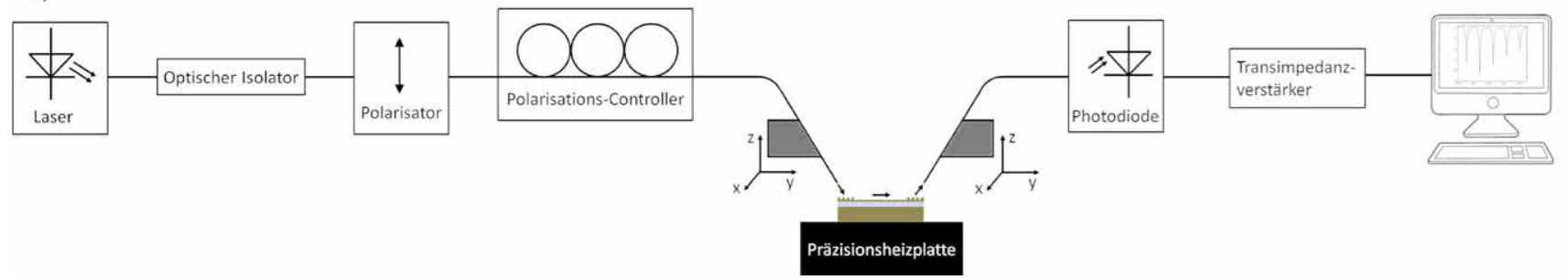

Abb. 3) Experimenteller Aufbau zur optischen Charakterisierung Chip-integrierter photonischer Bauelemente. Das Licht eines durchstimmbaren Lasers bzw. einer Superlumineszenzdiode wird über optische Fasern und ein Koppelgitter in den Chip ein- und ausgekoppelt. Zur Einstellung des Polarisationszustandes wird ein PolarisationsController genutzt und als Detektor dient eine rauscharme InGaAs-Photodiode. Der gesamte Aufbau befindet sich unter einer Flow-Box zur Vermeidung einer Kontamination der Chips. 
den zwei Kameras verwendet, um optimale Verhältnisse für das Ein- und Auskoppeln des Laserlichts in den Chip zu erreichen. Das in Abb. 4 gezeigte Spektrum eines teilweise geschlitzten Ringresonators wurde mit einem optischen Spektrenanalysator (Optical Spectrum Analyzer - OSA) aufgenommen. Das erhaltene Spektrum zeigt extrem scharfe Peaks, die sehr schmalbandig sind und einen großen Dämpfungsfaktor besitzen. Zur hochgenauen Charakterisierung der Transmissionspeaks wird ein durchstimmbarer Laser verwendet. Zur Detektion des Laserlichts wird dabei eine rauscharme Avalanche Photodiode mit hoher Verstärkung statt eines OSA verwendet.

Um die Messungen durchführen zu können, wurde der Schutzlack der Chips in einem Aceton-Bad entfernt. Der Schutzlack war in einem zusätzlichen Prozessschritt aufgebracht worden, um die Chips ohne Beeinträchtigung der Photonik-Strukturen vereinzeln zu können. Anschließend erfolgte die Beschichtung mit elektrooptischen Polymeren mittels SpinCoatings. Um sicherzustellen, dass kein Lösungsmittel in der Polymermatrix gebunden war, wurden die Chips nach dem Spin-Coating in einem Vakuumofen bei $90{ }^{\circ} \mathrm{C}$ über Nacht ausgeheizt. Als Lösungsmittel diente bei allen Experimenten 1.1.2.2-Tetrachlorethan. Für die ersten Experimen- te wurde ein in der Verarbeitung und Handhabung einfaches Polymersystem gewählt, welches aus kommerziell erhältlichen, preiswerten Materialien besteht und für Voruntersuchungen qualitativ ausreichende lineare und nichtlineare optische Eigenschaften aufweist. Diese Polymerkomposite bestanden aus einer Polymermatrix, Polymethylmethacrylat (PMMA) und dem - typischerweise mit einer Masse-Konzentration von 1 wt\% zugemischten - nichtlinearen optischen Chromophor Dispersrot 1 (DR1).

Nach dem Befüllen der Schlitzwellenleiter-Strukturen mit dem Wirt-GastPolymersystem PMMA/DR1 erfolgte die elektrooptische Charakterisierung der Ringresonatoren auf dem in Abb. 3 dargestellten Messplatz. Die aktive Messung erfolgte mit demselben Messaufbau, wie die passive Vermessung. Zur Ansteuerung dienten die GSG-Elektroden, die über Mikrokontaktspitzen mit einem Signalgenerator verbunden worden. Um Temperaturdrifts zu vermeiden wurde der Chip mit einer Präzisionsheizplatte auf eine Temperatur von $35{ }^{\circ} \mathrm{C}$ geheizt und stabilisiert. Das Anlegen einer Gleichspannung von 0 $\mathrm{V}$ bis $7 \mathrm{~V}$ verursachte eine erstaunlich hohe Resonanzwellenlängenverschiebung $\Delta \lambda$ von mehr als 0,5 nm. Zum Vergleich: Herkömmliche optische Schalter, basierend auf Silizium, erreichen Resonanzwellenlängenverschiebungen von bis zu 0,016 nm (Xuan et al. 2014).

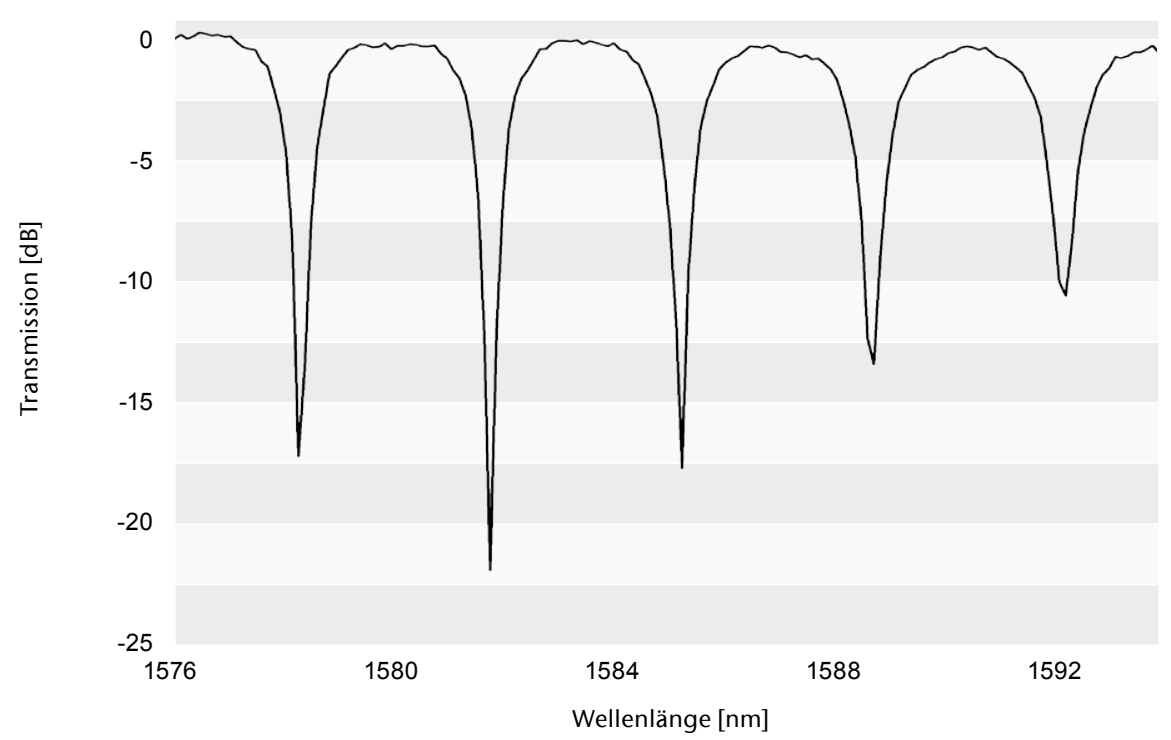

Abb. 4) Transmissionsspektrum eines partiell geschlitzten Ringresonators der mit einem Polymersystem bestehend aus PMMA und DR1 bedeckt ist. Das Spektrum zeigt extrem schmalbandige Peaks, wodurch derartige Resonatoren insbesondere für aktive photonische Bauelemente wie elektrooptische Modulatoren mit geringem Energiebedarf geeignet sind.

\section{Schlussfolgerungen und Ausblick}

Der Einsatz Chip-integrierter photonischer Bauelemente in schnellen optischen Datenübertragungsstrecken besitzt ein sehr großes Marktpotential, da der Bedarf an schnellen Datenübertragungsstrecken insbesondere für kurze und mittlere Übertragungslängen stetig wächst. Die hier gezeigten Ergebnisse, insbesondere die hohe Resonanzwellenlängenverschiebung und die schmalbandigen Resonanzpeaks des Ringresonators, zeigen, dass die SOH-Photonik für energieeffiziente Bauelemente in zukünftigen optischen Datenübertragungsstrecken interessant ist. Die SOH-Photonik ermöglicht funktionsoptimierte Lichtmodulatoren für eine energieeffiziente und hochbitratige Datenübertragung. Grund dafür sind günstige Synergieeffekte, da Vorteile der siliziumbasierten Chiptechnologie mit denen von funktionalen, organischen Materialien verknüpft werden.

Zukünftige Arbeiten sollten sich nunmehr mit der Integration von elektrooptischen Polymeren in die bestehende CMOS-Technologie beschäftigen. Zwar zeigen die hier demonstrierten Ergebnisse hervorragende Eigenschaften der SOH-Ringresonatoren, jedoch ist noch keine Langzeitstabilität nachgewiesen. Als besondere Herausforderung wird dabei die Verkapselung der Polymere angesehen. Ziel sollte es sein, äußere Einflüsse wie Temperatur, Luftfeuchtigkeit und UV-Licht zu reduzieren, um so die SOH-Photonik für den Konsumentenmarkt attraktiv zu machen. 


\section{LITERATUR}

Alloatti L, Palmer R, Diebold S, Pahl KP, Chen B, Dinu R, Fournier M, Fedeli J-M, Zwick T, Freude W, Koos C, Leuthold I (2014) $100 \mathrm{GHz}$ silicon-organic hybrid modulator. Light Sci Appl 3(5):e173. doi: 10.1038/ Isa. 2014.54

Almeida VR, Xu Q, Barrios CA, Lipson M (2004) Guiding and confining light in void nanostructure. Opt Lett 29(11):1209-1211. doi: 10.1364/OL.29.001209 Dalton LR, Sullivan PA, Bale DH (2010) Electric field poled organic electro-optic materials: state of the art and future prospects. Chem Rev 110(1):25-55. doi: $10.1021 / \mathrm{cr} 9000429$

Gould M, Baehr-Jones T, Ding R, Huang S, Luo J, Jen AK-Y, Fedeli J-M, Fournier M, Hochberg M (2011)

Silicon-polymer hybrid slot waveguide ring-resonator modulator. Opt Express 19(5):3952-3961. doi: 10.1364/ OE.19.003952

Korn D, Jazbinsek M, Palmer R, Baier M, Alloatti L, Yu H, Bogaerts W, Lepage G, Verheyen P, Absil P, Guenter P, Koos C, Freude W, Leuthold J (2014) Electro-Optic Organic Crystal Silicon High-Speed Modulator. IEEE Photonics ] 6(2):1-9. doi: 10.1109/JPHOT.2014.2314113 Miller SE (1969) Integrated Optics. An Introduction. Bell System Technical Journal 48(7):2059-2069. doi: 10.1002/j.1538-7305.1969.tb01165.x

Steglich P, Mai C, Stolarek D, Lischke S, Kupijai S, Villringer C, Pulwer $S$, Heinrich F, Bauer I, Meister $S$, Knoll D, Casalboni M, Schrader S (2015a) Novel Ring Resonator Combining Strong Field Confinement With High Optical Quality Factor. IEEE Photon Technol Let 27(20):2197-2200. doi: 10.1109/LPT.2015.2456133

Steglich P, Mai C, Stolarek D, Lischke S, Kupijai S, Villringer C, Pulwer S, Heinrich F, Bauer J, Meister S, Knoll D, Casalboni M, Schrader S (2016a) Partially slotted silicon ring resonator covered with electro-optical polymer. In: Vivien L, Pavesi L, Pelli S (eds), Sunday 3 April 2016, Brussels, Belgium. SPIE Proceedings. SPIE, 98910R. doi: $10.1117 / 12.2217725$

Steglich P, Padilla Michel Y, Villringer C, Dümecke S, Bauer J, Heinrich F, Casalboni M, Schrader S (2015b) Design Optimization of Slot-Waveguides Covered with Organic Cladding Materials for Integrated Photonic Devices. In: Knaut M (ed) 16. Nachwuchswissenschaftlerkonferenz. Tagungsband, 16 Apr 2015. Berliner Wissenschafts-Verlag, Berlin, ISBN: 978-3-8305-2044-3, pp 192-198

Steglich P, Villringer C, Dümecke S, Padilla Michel Y, Casalboni M, Schrader S (2015c) Silicon-on-Insulator Slotwaveguide Design Trade-offs. In: Proc PHOTOPTICS - 3rd Int Conf Photonics, Optics and Laser Technology, 12-14 Mar 2015, Berlin, ISBN: 978-989-758-093-2, pp 47-52. doi: 10.5220/0005336200470052

Steglich P, Villringer C, Pulwer S, Bauer J, Heinrich F, Casalboni M, Schrader S (2016b) Advanced Nanophotonics: Silicon-Organic Hybrid Technology. Wiss Beitr TH Wildau 20:45-48. doi: 10.15771/0949-8214_2016_1_6

Steglich P, Villringer C, Pulwer S, Casalboni M, Schrade $S$ (2016c) Design Optimization of Silicon-on-Insulator Slot-Waveguides for Electro-optical Modulators and Biosensors. In: Ribeiro PA, Raposo M (eds) Photoptics 2015 Proceedings of the 3rd International Conference on Photonics, Optics and Laser Technology, 1st ed. 2016. Springer Proceedings in Physics, vol 181. Springer International Publishing; Springer, Cham, ISBN: 3319301357 pp 173-187

Xuan Z, Ma Y, Liu Y, Ding R, Li Y, Ophir N, Lim AE-J, Lo G-Q, Magill P, Bergman K, Baehr-Jones T, Hochberg M (2014) Silicon microring modulator for $40 \mathrm{~Gb} / \mathrm{s}$ NRZ-OOK metro networks in O-band. Opt Express 22(23):28284-28291. doi: 10.1364/OE.22.028284

\section{AUTOREN}

Patrick Steglich (M.Eng.)* Claus Villringer (M.Eng.) Silvio Pulwer (M.Eng.)* Dipl.-Chem. Birgit Dietzel Dr. Viachaslau Ksianzou

Prof. Dr. Sigurd Schrader

Fachbereich Ingenieur- und Naturwissenschaften

Technische Hochschule Wildau

* Department of Industrial Engineering University of Rome "Tor Vergata"

E-Mail für Korrespondenz: patrick.steglich@th-wildau.de

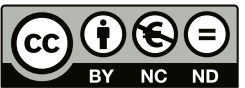

